\title{
SENSORS BASED ON THE GMI EFFECT IN FERROMAGNETIC MICROWIRES AND THEIR APPLICATIONS
}

\author{
Eva KOMOVÁ*, Rastislav VARGA**, Filip ŠIDÍK*, Žofia BARLÍKOVÁ* \\ *Department of Aerodynamics and Simulations, Faculty of Aeronautics, Slovak Republic \\ Technical University of Košice, Rampová 7, 04121 Košice, Slovak Republic, tel. +421 0907593 583, e-mail: eva.komova@tuke.sk \\ Institute of Physics, Faculty of Science, UPJŠ, Park Angelinum 9, 04154 Košice, Slovak Republic
}

\begin{abstract}
Ferromagnetic amorphous glass-coated microwires are progressive materials that exhibit very useful behaviour, like the giant magnetoimpedance (GMI) effect, which is the base the operating principles for different magnetic sensors. In order to obtain large GMI values, it is necessary to choose the optimal chemical composition of the wire material with large permeability and small penetration depth. Aim this work is study of the magnetic properties of $\mathrm{Co}_{70,5} \mathrm{Fe}_{4,5} \mathrm{Si}_{15} \mathrm{~B}_{10}$ alloys for GMI sensor application. Low prices and high flexibility of GMI sensors will warrant wide-ranging application in the near future.
\end{abstract}

Keywords: ferromagnetic amorphous alloy, glass-coated microwire, GMI effect

\section{INTRODUCTION}

Recent tendencies towards miniaturization of modern magnetic sensors and devices have stimulated development of magnetic materials with reduced dimensionality. Among these modern magnetic materials a family of glass-coated microwires have gained importance within the last few years. These materials exhibit a number of unusual magnetic properties. Many industrial and engineering applications for these materials have been proposed because of these properties.

Sensors made of these materials can be used to detect passage of moving object, simply by attaching small permanent magnets onto their surface. Detection is then observed as a decrease in the ac voltage on the microwire's ends. Such devices could be used in many industrial or traffic processes, using microwire's simplicity and low cost of production. These monitoring and control sensors may eliminate some errors of today's optical devices such as lower accuracy in environments with reduced visibility. Recently, a so-called nano-GMI sensor has been developed, and has been used in an antilock brake system (ABS) and for the measurement of electric injector speeds for the automotive and truck industries [1].

Use of flux gate (FG) sensor compasses, which are nowadays in many electronic devices such as cars, small boats and mobile phones, are no longer adequate for modern demands, especially because of their large size and high power consumption. These problems may be overcome by introducing GMI sensors into electronic compasses. When compared to FG sensors, GMI sensors have much reduced size and power consumption.

The increased flow of everyday traffic in the last decade is reflected in higher amount of accidents and traffic jams. This is partially due to the lack of automatic control and monitoring systems. Even though many systems, such as ultrasonic and video cameras, are being used for monitoring traffic, they may not be always suitable in certain conditions. Recently, however, a new use of amorphous wire GMI sensors has been proposed in car sensing systems. It's composed of a microwire built into a disk set on the road. In this device, the GMI sensor detects stray fields from the car body as it passes above the disk [2]. The length and speed of the car can be estimated by processing the signals from two GMI sensors. With a connected computer, this system can record length, velocity and time for about two thousand cars. Advantages as easy installation, insensitivity to weather and high reliability also favour this technique.

GMI sensors can not only be applied in road transport but also at the airports. Landing of an airplane is a significant source of potentially deadly incidents at the airport. If we consider the amount of activities carried out by a pilot, density of air traffic and other factors such as weather and the possibility of a technical failure, the probability of an unexpected event increases. GMI sensors can help operators of the airport to lower the possibility of an accident. The system consists of two sections: microwires embedded into runway surface, connected to microcomputers to detect changes of magnetic field, and a permanent magnet attached to undercarriage of an airplane. This requires minimal modifications on the vehicles. Movement of an airplane automatically changes the intensity of magnetic field, what can be detected on the sensors closest to it. Even exact types of airplanes can be determined by using different magnitudes of changes in magnetic field.

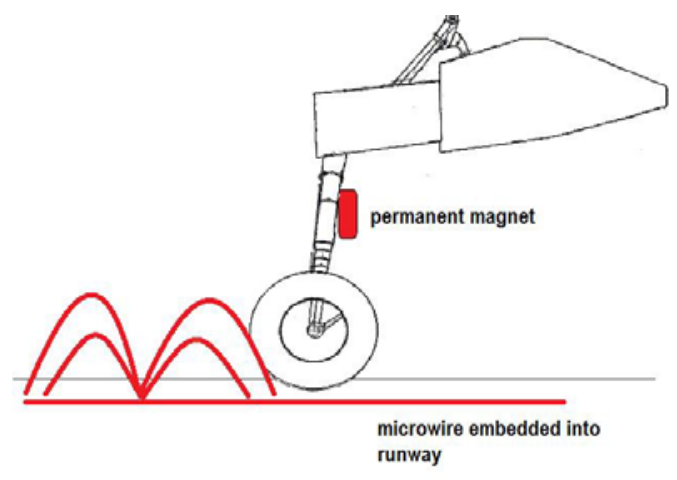

Fig. 1 Illustration of airplane detection system at the airport

Glass coated microwires and GMI sensors have many other perspective uses in biomedicine, information technologies, industry and space research. 
Thus, the positive magnetostriction compositions exhibit rectangular hysteresis loops, while the best magnetic softness is observed for the nearly-zero magnetostriction composition. Their main technological interest is related to the magnetic softness in nearly-zero magnetostriction composition, magnetic bistability in nonzero magnetostriction compositions and GMI effect [3].

\section{PRESENTATION OF THE GMI EFFECT}

When soft magnetic conductor is subjected to a small alternating current $I_{a c}$ a large change in the complex impedance $Z$ of the conductor can be achieved upon applying a magnetic field $H$. This is known as the giant magnetoimpedance (GMI) effect [4, 5].

Complex impedance $Z=R+j \omega L \quad(R$ and $L$ are resistance and inductance, respectively) of a magnetic conductor is given by the ratio $U_{a c} / I_{a c}$, where $I_{a c}$ is the amplitude of a sinusoidal current passing through the conductor and $U_{a c}$ is the voltage measured between the ends of the conductor. For a cylindrical ferromagnetic conductor it was obtained [1]:

$$
Z=R_{d c} k r \frac{J_{0}(k r)}{J_{1}(k r)},
$$

where $J_{0}$ and $J_{1}$ are the Bessel functions of the first kind, $r$ is the radius of the wire, $R_{d c}$ is the electrical resistance for a direct current and $k=(1+j) / \delta_{m}$ with imaginary unit $j, \delta_{m}$ is the penetration depth in a magnetic wire, with circumferential permeability $\mu_{\Phi}$ :

$$
\delta_{m}=\sqrt{\frac{\rho}{\pi f \mu_{\Phi}}},
$$

where $f=\omega / 2 \pi$ is the frequency of the alternating current, $I_{a c}$.

According to equations (1) and (2), the GMI effect can be understood as a consequence of the increase of the skin depth until it reaches the radius of the wire $r$ through the decrease of the circumferential permeability, $\mu_{\Phi}$. In order to obtain large GMI values, it is necessary to reduce skin depth by choosing magnetic materials that have large $\mu_{\Phi}$ and small $\delta_{m}$. The ideal magnetic properties, such as large magnetic permeability, were observed in nearly zero magnetostrictive Co-based alloys. Glass-coated amorphous microwires of Co-based composition have a unique circular magnetic anisotropy due to coupling between negative magnetostriction and frozen-in stress. Such anisotropy is important to realize large and sensitive magnetoimpedance effect, which is observed at frequencies when the skin effect is strong.

According to the frequency range, GMI effect can be classified into three regions $[1,6]$ : a/ Low-frequency regime (up to a few $\mathrm{kHz}$ )

The changes in voltage at the sample's ends are mainly due to the so-called magnetoinductive effect. The skin effect is very weak in this case. The change in the impedance of the sample upon application of the applied field results mainly from the contribution of inductance, which is proportional to the circumferential permeability.

b/ Intermediate frequency regime (between $100 \mathrm{kHz}$ and a few $\mathrm{MHz}$ )

GMI originates mainly from the variation of the skin depth due to strong changes of the effective magnetic permeability caused by the applied dc magnetic field. At frequency range of hundred $\mathrm{kHz}$ to $\mathrm{MHz}$, the change of impedance is mainly due to circular domain wall dynamics. At frequency range from few $\mathrm{MHz}$ to hundred $\mathrm{MHz}$, the domain wall motion is strongly damped and the rotational processes mainly contribute to the ac permeability and impedance change.

c/ High-frequency regime (several $\mathrm{MHz}$ up to $\mathrm{GHz}$ ), where the origin of GMI is believed to be related to the gyromagnetic effect and ferromagnetic relaxation. The maxima in GMI profiles are shifted towards higher fields, where the samples are already saturated magnetically [7].

\section{AMORPHOUS GLASS-COATED MICROWIRES}

Amorphous metallic alloys can be produced by a variety of rapid solidification processing techniques. The amorphous glass-coated microwires are usually made by so called Taylor-Ulitovsky melt spinning method [8]. A schematic illustration of this method is presented in Fig. 2.

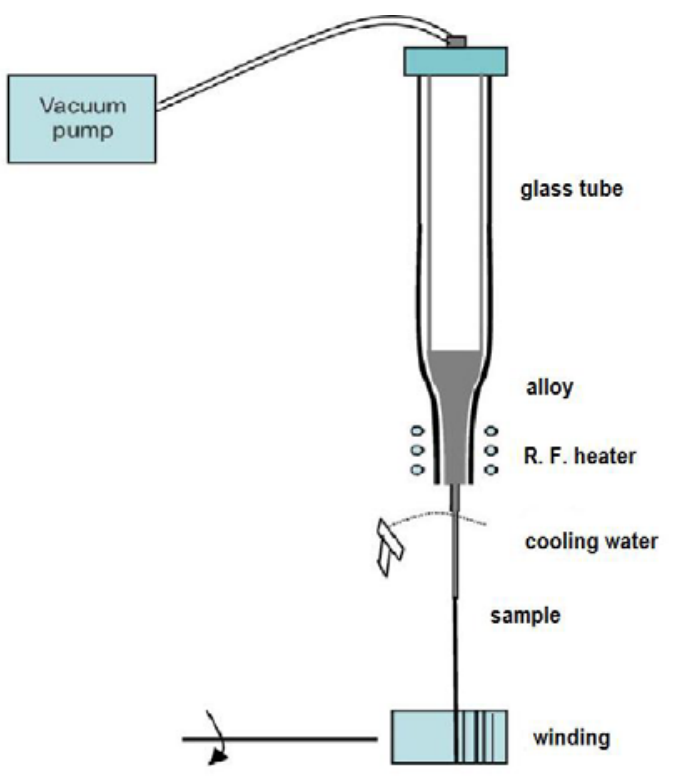

Fig. 2 Illustration of Taylor-Ulitovsky melt spinning method [8]

Final product is glass-coated microwire (Fig. 3) with metallic core diameter between $0,8-30 \mu \mathrm{m}$ and with glass coating thickness of 2-15 $\mu \mathrm{m}$. 


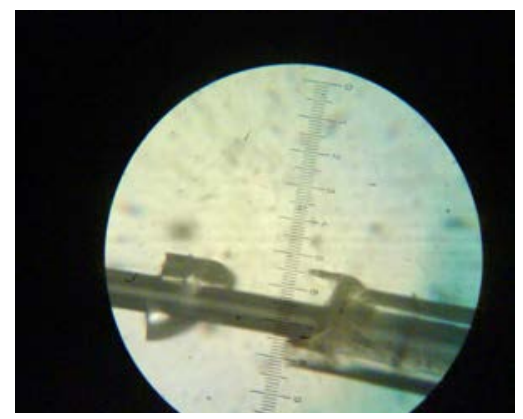

Fig. 3 Amorphous glass-coated $\mathrm{Co}_{70,5} \mathrm{Fe}_{4,5} \mathrm{Si}_{10} \mathrm{~B}_{15}$ microwire

The function of the glass insulation is twofold: the first is that it can protect the metallic core from oxidation and make it possible to fabricate the microwire of very small diameter. The second is that the glass coat provides more freedom degree to control the magnetic structure and properties of the wires.

\section{EXPERIMENT}

Our amorphous microwires with nominal composition $\mathrm{Co}_{70,5} \mathrm{Fe}_{4,5} \mathrm{Si}_{15} \mathrm{~B}_{10}$ were prepared in Instituto de Sciencia Materiales, Spain. The sample was $4 \mathrm{~cm}$ long, with the total diameter of $9 \mu \mathrm{m}$ and diameter of metallic parts of 7 $\mu \mathrm{m}$.

The field dependence of the impedance $Z$ has been measured by four-point method at room temperature (Fig. 4).

Electrical voltage $U_{a c}$ was estimated using oscilloscope. Values of the impedance $Z$ has been calculated from $U_{a c}$ and $I_{a c}$.

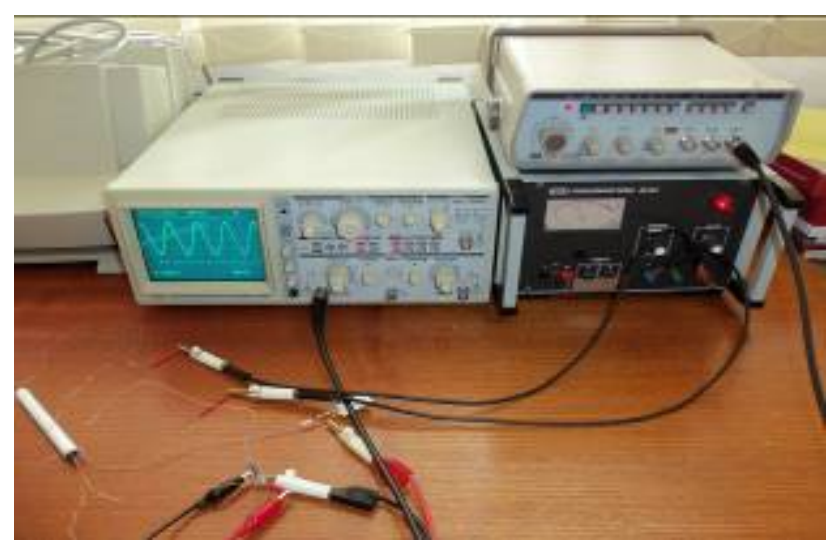

Fig. 4 Experimental workplace for GMI measurement

The magnetoimpedance ratio $\Delta Z / Z$ was calculated from the formula:

$$
\frac{\Delta Z}{Z}(\%)=\frac{Z(H)-Z\left(H_{\max }\right)}{Z\left(H_{\max }\right)} .100 \%,
$$

where $Z(H)$ and $Z\left(H_{\max }\right)$ are the impedance of the microwire in the measured external magnetic field and maximum magnetic field to saturate the wire, respectively. The dependences of the relative impedance $\Delta Z / Z$ on the applied external magnetic field were investigated for different frequency of the sinusoidal current $f=1,8 \mathrm{MHz}$, $2 \mathrm{MHz}$ and 2,2 $\mathrm{MHz}$. Measurement was made during increasing and decreasing applied $d c$ magnetic field, $H$.

\section{RESULTS AND DISCUSSION}

Figure 5 shows that GMI in glass-coated $\mathrm{Co}_{70,5} \mathrm{Fe}_{4,5} \mathrm{Si}_{10} \mathrm{~B}_{15}$ microwire depends on measuring frequency and achieves 19\%, 23\% and $24 \%$ for frequencies 1,8 MHz, $2 \mathrm{MHz}$ and 2,2 $\mathrm{MHz}$. In this frequency region, the skin effect in GMI is dominant and at higher $f$ the higher value $[\Delta Z / Z(\%)]_{\max }$ was found.

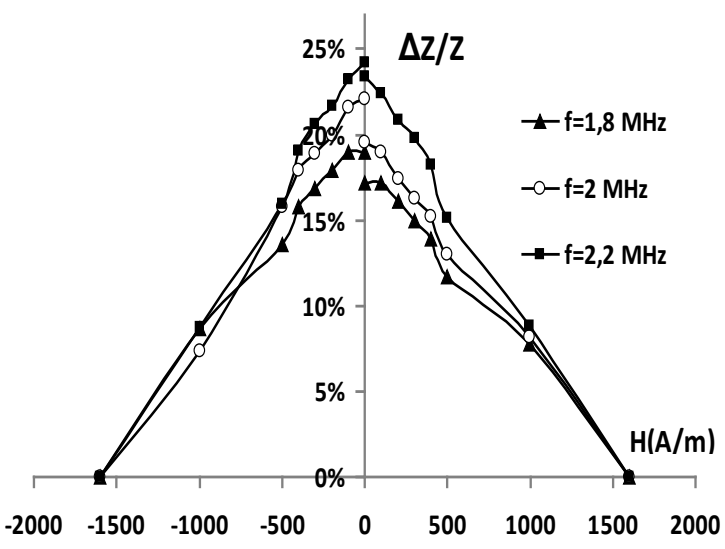

Fig. 5 Influence of varying frequency on the GMI effect ( $f=1,8 \mathrm{MHz}, 2 \mathrm{MHz}$ and 2,2 MHz)

In accordance with eq. (2), the magnetoimpedance increases with frequency because the impedance is proportional to $\left(f . \mu_{\Phi}\right)^{-1 / 2}$.

It is seen in Fig. 4, in all three cases, a small asymmetry in the GMI curve with respect to increasing and decreasing external magnetic field $H$. The origin of this hysteretic behaviour can be related to the hysteresis of the magnetisation process and to the spatial distribution of magnetic anisotropy, which determines the domain structure in amorphous ferromagnetic microwires.

\section{CONCLUSIONS}

We found that $\mathrm{Co}_{70,5} \mathrm{Fe}_{4,5} \mathrm{Si}_{10} \mathrm{~B}_{15}$ amorphous microwire exhibits GMI effect. The magnitude of GMI and its sensitivity varies with measuring frequency and achieved values $19 \%, 23 \%$ and $24 \%$ for frequencies $1,8 \mathrm{MHz}, 2$ $\mathrm{MHz}$ and 2,2 MHz. The maximum value of GMI effect experimentally observed is much smaller than the theoretically predicted value. The special thermal treatment is necessary to further improve the GMI in $\mathrm{Co}_{70,5} \mathrm{Fe}_{4,5} \mathrm{Si}_{10} \mathrm{~B}_{15}$ microwires.

Small hysteretic GMI behaviour was observed.

\section{ACKNOWLEDGMENTS}

This work was supported by the Slovak Ministry of Education Grant VEGA 1/0076/09, Grant APVV-0266-10 and APVV 0027-11.

We acknowledge M. Vazquez for sample preparation. 


\section{REFERENCES}

[1] PHAN, M. H. - PENG, H. X.: Progress in Materials Science 53, pp. 323-420, 2008.

[2] UCHIYAMA, T. - MOHRI, K. - ITHO, H. NAKASHIMA, K. - OHUCHI, J. - SUDO, Y.: IEEE Trans. Magn. 36, 3670-2, 2000.

[3] ZHUKOVA, V. - IPATOV, M. - ZHUKOV, A.: Sensors 9, pp. 9216-9240, 2009.

[4] PANINA, L. V. at all: IEEE Trans. Magn. 31, p. 1249, 1995.

[5] VAZQUEZ, M. - ZHUKOV, A. - GARCIA, K. L. PIROTA, K. R. - RUIZ, A. - MARTINEZ, L. KNOBEL, M.: JMMM 258-59, pp. 151-157, 2003.

[6] QIN, F. X. - PANKRATOV, N - PENG, H. X. TANG, J. - PANINA, L. V.: 17th International Conference on Composite Materials, 27-31 July, 2009.

[7] VAZQUEZ, M. - HERNANDO, A.: J.Phys. D: Appl. Phys., 29 (4), pp. 939-949, 1996.

[8] PARKHACHEV, V. N.: US patent 3,256,584.

Received November 27, 2012 , accepted February 9, 2013

\section{BIOGRAPHIES}

Eva Komová is working as an Associate Professor at Department of Aerodynamics and Simulations, Faculty of Aeronautics, Technical University in Kosice. She graduated from the Faculty of Sciences of University of P. J. Safarik, Kosice, in 1979 and received the $\mathrm{PhD}$ degree in experimental physics in 1999. Her scientific research is focusing on magnetic properties of amorphous and nanocrystalline soft magnetic alloys.

Rastislav Varga is an Associate Professor at Institute of Physics, Faculty of Sciences, UPJS in Kosice. He studied at Faculty of Sciences, UPJS in Kosice, where he obtained PhD degree in 1999. The main objects of his studies are physical properties of amorphous ferromagnetic microwires and its applications in different sensor devices. He is a member of EPS, published more than 70 papers.

Filip Šidík is a student at Faculty of Aeronautics, Technical University in Kosice. His research is dedicated to the GMI effect in ferromagnetic materials.

Žofia Barlíková is a student at Faculty of Aeronautics, Technical University in Kosice. She is devoted to the study of applications of modern magnetic materials in aviation. 